\title{
Passive Leg Raising for Fluid Responsiveness in Children: Is it Reliable?
}

\author{
Javed Ismail $^{1}$, Arun Bansal ${ }^{2}$ \\ Indian Journal of Critical Care Medicine (2020): 10.5005/jp-journals-10071-23430
}

Intravenous fluid administration is an integral part of management for critically ill children with impaired perfusion. Loss of more than $15-20 \%$ of intravascular volume impairs the preload which in turn results in decreased cardiac output, clinically manifesting with signs of impaired perfusion or shock. In children, major causes of shock, such as diarrhea, hemorrhage, sepsis, or anaphylaxis, result in either actual or relative intravascular hypovolemia (due to vasodilation). Rapid administration of fluid bolus could augment preload to improve the cardiac output and, thus, can potentially reverse shock. However, studies demonstrate that only half of the patients are preload responsive. In the other half, this fluid accumulates and leaks into the interstitial space of almost all organs and can result in serious adverse effects. Fluid leak is exacerbated in inflammatory states such as sepsis; and about $95 \%$ of the given fluid leaks out within 1 hour even among the fluid responders, thus limiting the beneficial effect. ${ }^{1}$ Hence, to identify which patient would respond to fluid bolus, tests of fluid responsiveness are recommended. Static parameters such as heart rate, systolic blood pressure, central venous pressure, and pulmonary artery occlusion pressure are the points of assessments and are poor predictors of fluid responsiveness. Dynamic parameters are based on heart-lung interaction and are proven to be reliable. Some of the common dynamic parameters are:

- Pulse pressure variation

- Stroke volume variation

- Pulse plethysmographic index

- Changes in aortic or carotid blood flow velocity with respiration

- Changes in inferior vena cava diameter with respiration

- Ventilator maneuvers such as end expiratory occlusion, tidal volume challenge

- Fluid challenge such as passive leg raising test (PLR) or mini fluid bolus.

Of these, fluid challenge is the gold standard, in which about $10-20 \mathrm{~mL} / \mathrm{kg}$ of crystalloid is administered and the real-time changes in cardiac output are measured. The PLR mimics fluid challenge, where the change in position increases the preload. The PLR can be used in both spontaneously breathing and ventilated patients (irrespective of the mode of ventilation or tidal volume) and in those with right heart failure or those who have postcardiac surgery with open sternum. In adults, a meta-analysis of 23 trials, PLR has proved to be accurate with area under the receiveroperating characteristic curve (AUC-ROC curve) of $0.95(95 \% \mathrm{Cl}$, $0.92-0.98)$ with a sensitivity of $86 \%(95 \% \mathrm{Cl}, 79-92)$ and specificity of $92 \%(95 \% \mathrm{Cl}, 88-96){ }^{2}$

In the study by El-Nawawy et al. $^{3}$ published in this issue of journal, 57 critically ill children were assessed for fluid responsiveness
${ }^{1}$ Pediatric Intensive Care, NMC Royal Hospital, Abu Dhabi, United Arab Emirates

${ }^{2}$ Department of Pediatrics, Advanced Pediatric Centre, Postgraduate Institute of Medical Education and Research, Chandigarh, India

Corresponding Author: Arun Bansal, Department of Pediatrics, Advanced Pediatric Centre, Postgraduate Institute of Medical Education and Research, Chandigarh, India, Phone: +91 9815455002, e-mail: drarunbansal@gmail.com

How to cite this article: Ismail J, Bansal A. Passive Leg Raising for Fluid Responsiveness in Children: Is it Reliable? Indian J Crit Care Med 2020;24(5):291-292.

Source of support: Nil

Conflict of interest: None

using PLR-induced hemodynamic changes estimated using transthoracic echocardiography against fluid challenge as the standard. Interestingly, the cohort consisted predominantly of infants with a mean age of 5 months, in contrast to the previous pediatric studies on PLR. ${ }^{4-6}$ For applicability of PLR in children, the caveat lies in the method of assessment of its response. In adults, Monnet et al. ${ }^{7}$ demonstrated that the highest value of change in outcome (aortic blood flow and the pulse pressure) was observed within the first 30 seconds of PLR. This implies that, to capture the flow changes accurately, a continuous real-time measurement tool is ideal. Commonly used techniques are esophageal Doppler aortic blood flow velocity, arterial pulse contour analysis, pulse pressure changes, and echocardiography.

In the index study, authors used transthoracic echocardiography to measure the changes in stroke volume $(\Delta \mathrm{SV})$ and cardiac index $(\triangle \mathrm{Cl})$ with $\mathrm{PLR}$; whereas, in other pediatric studies, cardiac output monitoring devices (NICOM, USCOM) have been utilized. ${ }^{4,6}$ As echocardiography is an operator-dependent technique, pilot validation with intraclass correlation of 0.93 [95\% confidence interval $(\mathrm{Cl})=0.91$ to $0.95, p<0.001$ ], implied good intraobserver reliability, thus adding confidence to the results. The positioning during PLR described by the authors is similar to that of adult and previous pediatric studies. ${ }^{4,6,7}$ However, positioning of young infants for PLR could be practically challenging. Authors reported that the change in stroke volume $(\triangle S V) \geq 10 \%$ during PLR indicates fluid responsiveness with AUC-ROC of $0.81(95 \% \mathrm{Cl}=0.68-0.9)$, a sensitivity of $65.38 \%$, and a specificity of $100 \%$. Also, the change in cardiac index $(\Delta \mathrm{Cl}) \geq 8.7 \%$ identified with AUC-ROC of $0.7(95 \%$ Cl $0.56-0.81$ ), sensitivity of $57.78 \%$, and a specificity of $91.67 \%$. This means that if $\Delta \mathrm{SV} \geq 10 \%$ and $\Delta \mathrm{Cl} \geq 8.7 \%$ on echocardiography during PLR, it is highly predictive of fluid responsiveness in children.

() The Author(s). 2020 Open Access This article is distributed under the terms of the Creative Commons Attribution 4.0 International License (https://creativecommons. org/licenses/by-nc/4.0/), which permits unrestricted use, distribution, and non-commercial reproduction in any medium, provided you give appropriate credit to the original author(s) and the source, provide a link to the Creative Commons license, and indicate if changes were made. The Creative Commons Public Domain Dedication waiver (http://creativecommons.org/publicdomain/zero/1.0/) applies to the data made available in this article, unless otherwise stated. 
In addition to the limitations acknowledged by the authors, our concern is that the echocardiography is highly operator dependent; and in spite of the repeated measurements by the same person during PLR and after the fluid bolus, there is an inherent risk of observer bias. Also, a specificity of $100 \%$ or a positive predictive value of 1 implies zero false positivity which indicated bias. Moreover, nonresponders account for a small proportion (only 5 of 57), and 3 of these 5 patients died, thereby limiting the generalizability of the results to other cohorts. Overall, this study adds to the limited data on fluid responsiveness in critically ill children. Future studies should in addition account for the growthrelated changes in ventricular compliance and its modifiers during disease states. Also, more reliable, cheaper, and easier automated devices to continually assess the cardiac output could pave the way for reliable results and better outcomes.

\section{References}

1. Long E, Babl FE, Oakley E, Sheridan B, Duke T, Pediatric Research in Emergency Departments International Collaborative. Cardiac index changes with fluid bolus therapy in children with sepsis-an observational study. Pediatr Crit Care Med 2018;19(6):513-518. DOI: 10.1097/PCC.0000000000001534.
2. Cherpanath TGV, Hirsch A, Geerts BF, Lagrand WK, Leeflang MM Schultz MJ, et al. Predicting fluid responsiveness by passive leg raising: a systematic review and meta-analysis of 23 clinical trials. Crit Care Med 2016;44(5):981-991. DOI: 10.1097/CCM.0000000000001556.

3. El-Nawawy AA, Farghaly PM, Hassouna HM. Accuracy of passive leg raising test in prediction of fluid responsiveness in children. Indian J Crit Care Med 2020;24(5):344-349.

4. Lu G, Yan G, Chen Y, Lu Z, Zhang L, Kissoon N. The passive leg raise test to predict fluid responsiveness in children - preliminary observations. Indian J Pediatr 2015;82(1):5-12. DOI: 10.1007/s12098-0131303-5.

5. Lukito V, Djer MM, Pudjiadi AH, Munasir Z. The role of passive leg raising to predict fluid responsiveness in pediatric intensive care unit patients. Pediatr Crit Care Med 2012;13(3):e155-e160. DOI: 10.1097/ PCC.0b013e3182388ab3.

6. Wu Y, Liu X, Li C, He Y, Yang W, Yang Y, et al. Clinical observation of non-invasive ultrasonic cardiac output monitor combined passive leg raising test in predicting the children volume responsiveness. Zhonghua Wei Zhong Bing Ji Jiu Yi Xue 2014;26(1):46-50. DOI: 10.3760/cma.j.issn.2095-4352.2014.01.010.

7. Monnet X, Rienzo M, Osman D, Anguel N, Richard C, Pinsky MR, et al. Passive leg raising predicts fluid responsiveness in the critically ill. Crit Care Med 2006;34(5):1402-1407. DOI: 10.1097/01. CCM.0000215453.11735.06. 